\title{
Niveles de resiliencia emocional y el aprendizaje virtual, en los estudiantes de la carrera de turismo - UTB
}

\author{
Emotional resilience levels and virtual learning in the tourism students in \\ the UTB
}

Christian Rivera García. ${ }^{1}$, Cecilia Cáneppa. ${ }^{2}$, Victoria Guijarro. ${ }^{3} \&$ Maricela Izurieta. ${ }^{4}$

\begin{abstract}
DOI: https://doi.org/10.33262/concienciadigital.v4i4.2.1979

Resistance, support and adaptability are the basis of the resilience that allows for activities with a high stress discharge in university learning processes. In higher education, resilience is a significant ability for gradual emotional change and transformation, which allows to expand the possibilities of the human being in the face of different difficult contextual realities. The objective of this research was to identify levels of emotional resilience to improve virtual learning in academic undergraduate vocational training activities. As a scenario for the application of this research, it was considered to carry out types of research such as: descriptive, exploratory, explanatory and correlational, using methods of analysis, synthesis, inductive, deductive, ethnographic, and critical partner in application of a questionnaire to conduct the survey of students from Tourism Career of the UTB in support of the survey applied to 203 participants were obtained as results that the group surveyed shows an average capacity of resilience, with emotional difficulties to face unexpected actions in their family and university life, in the field of virtual learning students have developed new strategies to learn from an investigative order, improving their expertise in the use of technological tools, participants have developed
\end{abstract}

\footnotetext{
${ }^{1}$ Universidad Técnica de Babahoyo FCJSE, Ecuador. crivera@utb.edu.ec

2 Universidad Técnica de Babahoyo FCJSE, Ecuador. ccaneppa@utb.edu.ec

3 Universidad Técnica de Babahoyo FCJSE, Ecuador. rguijarro@utb.edu.ec

${ }^{4}$ Universidad Técnica de Babahoyo FCJSE, Ecuador. eizurieta@utb.edu.ec
} 
synchronous activities creating new experiences, and emotional strengths, individual and group, these results demonstrate an interest in the acquisition of social and academic links, technology allows to regenerate the intuitive spirit of cutting-edge students in problem solving and decision making processes.

Keywords: virtual learning, strategies, contextual realities, emotional resilience.

Resumen: La resistencia, el soporte y la adaptabilidad son la base de la resiliencia que permite realizar actividades con una gran descarga de estrés en procesos de aprendizaje universitario. En educación superior, la resiliencia es una habilidad significativa de cambio y transformación emocional gradual, que permite ampliar las posibilidades del ser humano frente a diferentes realidades contextuales difíciles. En esta investigación se consideró como objetivo: identificar los niveles de resiliencia emocional para mejorar el aprendizaje virtual en actividades académicas de formación profesional de pregrado. Como escenario para la aplicación de esta investigación se consideró realizar tipos de investigación como: descriptivo, exploratorio, explicativo y correlacional, utilizando los métodos de análisis, síntesis, inductivo, deductivo, etnográfico, y socio crítico en aplicación de un cuestionario para realizar la encuesta a los estudiantes de la Carrera de Turismo de la UTB en apoyo a la encuesta aplicada a 203 participantes se obtuvieron como resultados que el grupo encuestado muestra una capacidad media de resiliencia, con dificultades emocionales para afrontar acciones inesperadas en sus vidas familiares, personales y universitarias, en el campo del aprendizaje virtual los estudiantes han desarrollado nuevas estrategias para aprender desde un orden investigativo, mejorando su experticia en la utilización de herramientas tecnológicas, los participantes han desarrollado actividades sincrónicas creando nuevas experiencias, $\mathrm{y}$ fortalezas emocionales, individuales y grupales, estos resultados demuestran un interés en la adquisición de nexos sociales y académicos, la tecnología permite regenerar el espíritu intuitivo de estudiantes de vanguardia en procesos de solución de problemas y toma de decisiones.

Palabras clave: aprendizaje virtual, estrategias, realidades contextuales, resiliencia emocional.

\section{Introducción}

El COVID-19 develó diferentes realidades sociales, económicas, ambientales, políticas, culturales, axiológicas y educativas insuperables psicológicamente a nivel planetario. Estas transformaciones conductuales de depresión, ansiedad, confrontaciones relacionales es el resultado de un espíritu atomizado por el manejo de la información, en los medios de comunicación, medios de producción y los patrones de consumo dentro del margen emocional humano. Realidad presente en la adversidad es la resiliencia, término mayormente utilizado en psicología la cual se enfoca en buscar los factores positivos que pueden inducir a una transformación que le permita al ser humano sobreponerse a las adversidades de carácter, familiar, social y profesional. La resiliencia en la educación 
constituye un soporte que refuerza la calidad y cualidad del estudiante, profesor y demás integrantes de la comunidad educativa en busca de la excelencia y superación de desafíos. El aprendizaje actual vinculado a contextos cada vez más innovadores que requieren una mayor y mejor predisposición de los involucrados en el proceso de enseñanza aprendizaje necesita estudiantes que desarrollen una actitud resiliente para afrontar los inconvenientes que se generan a partir de estos nuevos entornos educativos. El aprendizaje virtual es uno de los entornos, que, a raíz de la pandemia, se ha convertido en el elemento primordial en la educación a nivel mundial. Las clases virtuales desarrolladas dentro de un entorno digital, a decir de Santos et al. (2018) son un intercambio de información que comparte conocimientos, dudas, consultas y en el que se utiliza herramientas tecnológicas que facilitan la entrega de información. A pesar de las grandes ventajas que la virtualidad ha traído en un mundo amenazado por el distanciamiento, el encierro y que ha permitido continuar en una nueva realidad con las actividades diarias de trabajo y estudio, también ha generado un muevo tipo de problemática en especial para la educación del sector público que aún no estaba preparado para un cambio tan drástico hacia el aprendizaje virtual.

Según la UNESCO (2020), el hacinamiento impide contar con un espacio adecuado para estudiar y descansar, lo que repercute en el desarrollo cognitivo en la infancia y las trayectorias laborales y de bienestar en la adultez, a la vez que favorecen una mayor propensión a situaciones de abuso. Este es un contexto volátil para el proceso enseñanzaaprendizaje, derivado en funciones y roles complementarios en familia, que priva angustiosamente a cada miembro sobre su emancipación, obligatoria y voluntaria de esparcimiento, esta racionalidad humana se eslabona progresivamente en el constructo teórico de la resiliencia en tiempo real.

Así mismo la investigación de Aldana-Zavala \& Rodríguez-Partidas (2020), confirman la presencia de estrés, depresión y ansiedad en el área educativa, desembocando en un PEA con concepciones reduccionistas de intelectualidad programática estudiantil, los cuadros psicológicos no tardan en demostrar desequilibrio sistemático en la enseñanza de calidad.

A decir de Rojas (2015), la resiliencia permite la adaptabilidad a situaciones difíciles tomando como base el contexto cultural, académico, emocional, conductual y físico. El estudio de la resiliencia ha provocado diferentes contraposiciones entre los investigadores quienes apoyan la tesis que la resiliencia se ajusta a factores del entorno como el apoyo familiar y los ambientes de aprendizaje otros que basan en los factores personales como la autoestima, la motivación intrínseca y autosuficiencia que orienta a la resolución de problemas, sin embargo existe una conciliación entre ambos factores y se puede establecer que tantos los factores del entorno y los personales son un conjunto que permite al ser humano llegar a esta etapa de construcción que es la resiliencia.

La Resiliencia es un término que poco a poco se ha ubicado en los diferentes parámetros de la vida emocional del ser humano, cobra hoy más que nunca un valor preponderante en la superación de las dificultades que se enfrentan a una sociedad moderna. La 
resiliencia ha sido motivo de muchas investigaciones en diferentes campos como el psicológico se define como: "La Habilidad para resurgir de la adversidad, adaptarse, recuperarse y acceder a una vida significativa y productiva" (Silva et al., 2018, p.14). Lo cual indica que el ser resiliente es capaz de no solo superar sus dificultades sino desarrollar lo más posible sus potencialidades emocionales.

Coronel (2019), en sus investigaciones dentro del campo educativo consideran el término resiliente como sinónimo de invulnerable, invencible y resistente en la educación. Afirman que el proceso es de vida, donde la persona debe superar eventos de estrés y trauma sin quedar marcas de hostilidad, se vincula con la pedagogía por cuanto es la ciencia que enseña a vivir bien la vida superando los momentos difíciles.

Para Asch (2021), la resiliencia en educación conlleva a la recuperación y adaptación a la adversidad desarrollando una competencia social, académica y vocacional. Los estudios recientes realizados en el sector de educación básica y media superior en este campo determinan la importancia de la resiliencia en los estudiantes que desde pequeños se encuentran expuestos a diversos factores de riesgo que puede producirles vulnerabilidad sino tienen el manejo de los niveles de frustración manejos en sus estudios con interés y motivación.

Según Prado-Solís et al. (2017), mencionan que resiliencia y educación, dualidad conceptual inseparable muchos estudios se han efectuado donde se destaca la relevancia del rol de la escuela, el profesor y las experiencias escolares para la formación de la resiliencia en los estudiantes en donde el profesor es el generador de un ambiente de aprendizaje amable que motive a los estudiantes en su quehacer diario, de allí la importancia de una comunidad educativa con actores de conductas resilientes que provean apoyo cuando sea necesario.

Soldevilla et al. (2018), establecen que en un estudio realizado en Chile se encontró que los factores que impulsan la calidad de vida y salud mental de los estudiantes que los ayudan a superar la adversidad están ligados a la familia y la educación. Por lo que las instituciones educativas tienen por objetivo primordial el desarrollo de competencias sociales para que se desenvuelvan en el mundo moderno, así como conocer los factores de resiliencia que permitan una intervención positiva que la genere.

La resiliencia en educación permite al estudiante y demás integrantes de la comunidad educativa reforzar sus cualidades en el cual los factores protectores como la familia y la educación logran que se superen las adversidades y se avance en el proceso de aprendizaje no solo de conocimientos sino también en el desarrollo del factor individual como ser humano que está dispuesto a enfrentar y superar los obstáculos convirtiéndose en un ser social productivo.

El papel que desempeña el docente como guía y facilitador del proceso de enseñanza es vital por cuanto su resiliencia en el trato con los estudiantes motiva y crea en ellos la actitud de tomarlo como ejemplo para seguir sus pasos y aprender de su profesor no solo conocimiento sino también en conducta resiliente. 
Hasta hace poco la educación virtual estaba destinadas a ser parte complementaria de la educación presencial con estrategias de enseñanza y aprendizaje relacionadas con las TICS lo cual es un desafío para el rol del docente quien está llamado a ser quien aporte el conocimiento sobre el manejo de las nuevas herramientas que propician en los estudiantes capacidades informáticas en desempeño de su perfil profesional.

Esta nueva forma de aprendizaje según Bonilla (2016). la educación virtual es una educación a distancia que se lleva a efecto en el ciberespacio que aporta un escenario diferente para la comunicación entre el docente y estudiante.

Santos (2018) explica "Las clases virtuales son un entorno digital en el que se lleva a cabo un intercambio de información y conocimientos que tiene por objetivo proporcionar un aprendizaje entre los usuarios que participan en ella" Esta definición indica que es un entorno en el cual no solo se transmite una información, sino más bien es una interacción donde se despejan dudas y se mantiene un proceso evaluativo. Marciniak \& Sallán (2018), enmarcan a la educación virtual dentro de un ambiente donde los recursos tecnológicos sean los apropiados y el sistema utilizado permitan ofrecer una estructura y contenido virtual con un verdadero valor formativo.

Autores en diferentes investigaciones vinculadas al entorno virtual de enseñanza como Area \& Adell (2009), quienes enfatizan que las dificultades en el aprendizaje virtual están dadas por la calidad de enseñanza impartida por el medio virtual, los recursos didácticos utilizados que a decir de Lara (2001) se dividen en sincrónicos y asincrónicos y que se deben utilizar para una mayor productividad. Desde otro punto de vista Marciniak \& Sallán (2018), establece que los problemas que los estudiantes pueden afrontar en el campo virtual están vinculados a otros factores como la disponibilidad de los recursos tecnológicos y su calidad. Es importante destacar que un problema que afecta la enseñanza virtual es el acceso a los recursos tecnológicos como el internet.

En un estudio realizado por Lloyd (2020), con estudiantes universitarios se determinó que aquellos estudiantes sin acceso a los recursos tecnológicos se frustran trayendo como consecuencia un estado de ansiedad y stress que los puede llevar a la deserción. Estas investigaciones ponen de manifiesto que, aunque la educación virtual ha pasado de ser un complemento en el proceso educativo a una prioridad que se magnifico con la pandemia para mantener el sistema educativo funcionando también evidencio las deficiencias tecnológicas y desigualdades socioeconómicas entre una educación virtual pública y privada.

Huaman (2021), en una investigación realizada sobre las implicaciones del paso del aprendizaje presencial al aprendizaje virtual en tiempos de pandemia enfatiza que antes de la pandemia habían instituciones educativas que utilizaban herramientas tecnológicas con personal capacitado en el uso de las TIC a diferencia de las instituciones públicas que ponían poco cuidado en capacitar al personal docente en el manejo de la tecnología lo cual se hizo evidente al momento de tener que impartir las clases solo a través del medio virtual. En el conversatorio 
El trabajo de Huamán (2021), advierten que las emociones negativas como la depresión, soledad, ira, son contraproducentes para el buen desempeño profesional del docente, siendo conveniente a partir de lo planteado, estudiar como la resiliencia podría influir en superar los inconvenientes descritos anteriormente, así, como podría contribuir en el mejoramiento de los docentes desde una óptica personal, social, pedagógica. El hacinamiento impide contar con un espacio adecuado para estudiar y descansar, lo que repercute en el desarrollo cognitivo en la infancia y las trayectorias laborales y de bienestar en la adultez, a la vez que favorecen una mayor propensión a situaciones de abuso (UNESCO, 2020, p.14).

El aprendizaje virtual considera las formas más amplias de combinar: imágenes, textos, audios, gráficos, videos, tablas, entre otras, para garantizar la interacción de los componentes del proceso enseñanza-aprendizaje y mejorar la relación de docentes y estudiantes en propuesta de innovar la calidad de la enseñanza universitaria, este pensamiento holístico considera la adaptabilidad académica en tiempos de pandemia, nodos gordianos que facilitan la utilización de las plataformas virtuales en conjunto con las herramientas asincrónicas de tendencias actuales. El problema de aprendizaje se agudiza cuando la conectividad se aísla del desarrollo productivo académico, el mapa de lugares accesibles se complejiza por el relieve geográfico de territorio, y los escasos recursos económicos dilatan el conocimiento, en suma, hacia el estrés emocional se generaliza en los estudiantes universitarios, el contexto familiar y el personal.

\section{Metodología}

Se empleó el método analítico sintético y la técnica de análisis de contenido con la intención de proyectar una síntesis teórica sobre el tema, a partir de escrutar trabajos de investigación publicados en Pubmeb, Scielo, Scopus, Wos, Latindex 2.0, Redalyc, así como repositorios institucionales de organismos y universidades públicas - privadas, nacionales y extranjeras.

De ese modo, la investigación de revisión documental tiene por objetivo analizar la resiliencia emocional y el aprendizaje virtual del estudiante de la carrera de turismo en tiempo de pandemia. Para este estudio y para la medición del nivel de resiliencia en los estudiantes universitarios, se utilizó la información proporcionada por la comisión de tutorías y bienestar estudiantil de la carrera, y los informes de desempeño docente.

La escala de resiliencia construida fue considerando las propiedades psicométricas en una muestra de 203 estudiantes, concordando con la escala original, en el hecho de que la misma está integrada por tres dimensiones (1. Problemas académicos, 2. Dificultades emocionales con el distanciamiento social y 3. Desarrollo y formación de la resiliencia). Estas tres dimensiones toman en consideración las siguientes características de resiliencia: solución de problemas, toma de decisiones, dificultades integrales emocionales autoestima, aprendizaje virtual sincrónico, aprendizaje virtual asincrónico, perseverancia, confianza en sí mismo, satisfacción de necesidades 


\section{Resultados}

El enfoque cuantitativo permite realizar una investigación de carácter descriptivo, el impacto de la creación de micro emprendimientos momentáneos y limitados, sugiere un trato especial en la economía familiar, es un fenómeno escasamente estudiado que dirige las necesidades poblacionales a soluciones parches, con excepciones del manejo adecuado de las tecnologías de información y comunicación en el menor tiempo posible, estos emprendimientos posibilitan la generación de economía auxiliar, para la obtención de los resultados se solicitó llenar una encuesta dirigida a micro emprendedores de una zona específica, el muestreo intencional fue realizada a 100 personas emprendedoras, particularmente en negocios de alimentación.

\section{Tablas 1}

Niveles de resiliencia emocional y el aprendizaje virtual carrera de turismo

\begin{tabular}{|c|c|c|c|}
\hline $\begin{array}{c}\text { Indicadores dominantes } \\
\text { resiliencia }\end{array}$ & $\begin{array}{l}\text { Indicadores } \\
\text { dominantes } \\
\text { virtualidad }\end{array}$ & $\begin{array}{c}\text { Porcentaje de } \\
\text { aceptación prioritaria }\end{array}$ & $\begin{array}{c}\text { Acciones } \\
\text { procedimentales en } \\
\text { beneficio de la } \\
\text { creatividad e innovación }\end{array}$ \\
\hline Problemas académicos & Clases sincrónicas & $91 . \%$ & $\begin{array}{c}\text { Tutorías académicas } \\
\text { debería incluir equipo } \\
\text { informático }\end{array}$ \\
\hline $\begin{array}{c}\text { Dificultades emocionales } \\
\text { con el distanciamiento } \\
\text { social }\end{array}$ & $\begin{array}{c}\text { Tutorías y bienestar } \\
\text { estudiantil }\end{array}$ & $41.9 \%$ & $\begin{array}{c}\text { Orientación hacia el } \\
\text { control de la ansiedad y } \\
\text { miedo }\end{array}$ \\
\hline $\begin{array}{l}\text { Desarrollo y formación } \\
\text { de la resiliencia }\end{array}$ & $\begin{array}{l}\text { Complementariedad } \\
\text { aprendizaje } \\
\text { asincrónico }\end{array}$ & $48.5 \%$ & $\begin{array}{c}\text { Incrementar metodología } \\
\text { del trabajo autónomo }\end{array}$ \\
\hline
\end{tabular}

\section{Conclusiones}

- La inseminación del conocimiento en esta era digital permite el acceso rápido a la información y comunicación, desarrollo de habilidades tecnológicas no solo en el manejo de las herramientas virtuales, sino el manejo emocional de las dificultades que rodean el entorno de los milenials. Es en este punto que la resiliencia cobra una gran importancia para la adaptabilidad positiva a las adversidades que se pueden generar dentro de un aprendizaje que depende de sistemas virtuales que muchos profesores, estudiantes y más integrantes de la comunidad educativa apenas manejaban. Situación que se magnifico aún más en las instituciones educativas publicas donde la conectividad es escasa, y los docentes desconocían el manejo de plataforma y herramientas tecnológicas.

- La resiliencia y el aprendizaje virtual es un tema que amerita estudio por la necesidad de crear ambientes de aprendizaje con docentes que sean tutores de una enseñanza resiliente que provean una instrucción no solo de conocimientos sino de actitudes positivas proporcionadas a sus estudiantes para que sean los 
constructores y ejemplificadores de soluciones a las adversidades que se presenten.

- El impacto del presente estudio abarca al campo educativo en el que los estudiantes tendrán una mejor adaptabilidad entendida como una adaptación que construya soluciones y no una que sea conformista, en el campo profesional donde los graduados de las diferentes áreas de experticia ingresarán al campo laboral con una actitud asertiva y proactiva frente a los momentos de stress y de adversidad que aporte ideas productivas de superación individual y de equipo de trabajo.

- El proceso de enseñanza-aprendizaje dentro de la virtualidad requiere un aprendizaje autónomo y significativo donde el docente sea el tutor que posibilite no solo la transmisión de un conocimiento sino la posibilidad de construir actitudes positivas frente a los retos y desafíos a los cuales se enfrentan diariamente en un entorno con profundos limitantes y dificultades, es decir, conciliar un estado de resiliencia donde los actores del proceso educativo virtual se apoyen en la construcción de soluciones a las dificultades y generen actitudes de acompañamiento, comunicación y participación activa, que produzca un estado de resiliencia que origine una transformación positiva frente a las adversidades virtuales y del entorno en general.

- El proceso de enseñanza y aprendizaje en esta nueva normalidad requiere de un currículo de emergencia y complementario dispuesto a buscar la seguridad psicológica de los actores académicos, proporcionando confianza y apoyo sine qua non sobre la realización y alcance de objetivos y metas preestablecidas, significación práctica en la generación de nuevas políticas educativas que integren todos los niveles del sistema educativo ecuatoriano, mejorando, el clima académico para el estudiante con equipo tecnológico, internet fijo, impresora con escáner, cámara web, micrófono, espacio silencioso iluminación precisa, silla ergonómica, entre otras.

\section{Referencias bibliográficas}

Aldana-Zavala, J. J., \& Rodríguez-Partidas, N. J. (2020). Depresión, ansiedad y estrés por COVID-19 en actores educativos Depression, anxiety and stress due to COVID19 in educational actors.

Area, M., \& Adell, J. (2009). eLearning: Enseñar y aprender en espacios virtuales. Tecnología Educativa. La formación del profesorado en la era de Internet, 391-424.

Asch, J. M. (2021). El análisis de la resiliencia y el rendimiento académico en los estudiantes universitarios. Revista Nacional de Administración, 12(1), e3534e3534.

Coronel Quintero, L. (2019). La Resiliencia como un enfoque transformador dentro del acto pedagógico. 
Bonilla, L. A. G. (2016). Deliberación entorno a la Educación Virtual. Interconectando Saberes, (1), 77-89.

Huaman Ramos, L. (2021). Educación remota y desempeño docente en las instituciones educativas de educación secundaria en el distrito de Huancavelica en tiempos de Covid-19.

Lloyd, M. W. (2020). Desigualdades educativas y la brecha digital en tiempos de COVID19.

Marciniak, R., \& Sallán, J. G. (2018). Dimensiones de evaluación de calidad de educación virtual: revisión de modelos referentes. RIED. Revista Iberoamericana de Educación a Distancia, 21(1), 217-238.

Prado-Solís, M., Becerra-Quiñonez, W. V., \& Torres-Estacio, C. C. (2017). Resiliencia y educación, dualidad conceptual inseparable. Polo del Conocimiento, 2(1), 145161.

Rojas Flórez, L. F. (2015). Factors Affecting Academic Resilience in Middle School Students: A Case Study (Factores que Afectan la Resiliencia Académica en Estudiantes de Bachillerato). GIST Education and Learning Research Journal, 11, 63-78.

Santos, E. L., García-Medina, I., \& Bellido-Pérez, E. (2018). Comunicación y moda en el entorno digital: comportamiento del público seguidor de blogueras de moda./ANABEL/Downloads/3805-Texto\%20del\%20art\%C3\%ADculo-8656-110-20191226.pdf

Santos, Méndez, M. E. (2018). Introducción de un diseño de una plataforma virtual para la interacción entre docente y estudiante con la integración de un asistente virtual (Chatbot); orientada a los estudiantes del 2 do y 3 ro de bachillerato en la especialización de informática del colegio fiscal técnico Provincia De Bolivar (Doctoral dissertation, Universidad de Guayaquil. Facultad de Ciencias Matemáticas y Físicas. Carrera de Ingeniería En Networking y Telecomunicaciones).

Silva, G. S., Cordero, C. Z., \& Urdanivia, J. S. (2018). La resiliencia: un factor decisivo para el crecimiento y mejora de las organizaciones. Gestión en el tercer milenio, 20(39), 13-24.

Soldevilla, A. R., Hernández, J. A. D., \& Rodríguez, P. E. (2018). Resiliencia y rendimiento académico en estudiantes de educación superior. Revista Educretam, 1, 45-55.

UNESCO. (2020). La educación en tiempos de la pandemia de COVID-19 [Education in times of the COVID-19 pandemic]. http://hdl.handle.net/11362/45904 


\section{PARA CITAR EL ARTÍCULO INDEXADO.}

Rivera García, C., Cáneppa, C., Guijarro, V., \& Izurieta, M. (2021). Niveles de resiliencia emocional y el aprendizaje virtual, en los estudiantes de la carrera de turismo - UTB. ConcienciaDigital, $4(4.2)$

124-133. https://doi.org/10.33262/concienciadigital.v4i4.2.1979

\section{¿Ciencia}

El artículo que se publica es de exclusiva responsabilidad de los autores y no necesariamente reflejan el pensamiento de la Revista Conciencia Digital.

El artículo queda en propiedad de la revista y, por tanto, su publicación parcial y/o total en otro medio tiene que ser autorizado por el director de la Revista Conciencia Digital.

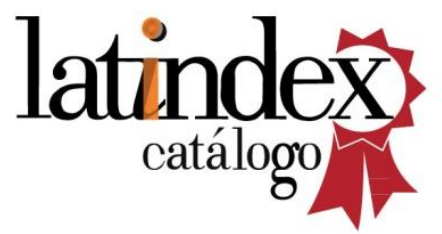

